\title{
Hacia una problematización geo- histórica y contextual del espacio urbano segregado
}

\author{
Para uma problematização geo-histórica e contextual do \\ espaço urbano segregado
}

Towards a geo-historical and contextual problem of the segregated urban space

Alexandra López Martínez [a] [D

[a] Tecnológico de Antioquia Institución Universitaria, Medellín, Colombia.

Cómo citar: López Martínez, A. (2021). Hacia una problematización geo-histórica y contextual del espacio urbano segregado. urbe. Revista Brasileira de Gestão Urbana, v. 13, e20200044. https://doi.org/10.1590/21753369.013.e20200044

\section{Resumen}

Considerando el caso específico de la ciudad de Ibagué, Colombia, este artículo muestra un análisis geohistórico y contextual que presenta los factores determinantes de la segregación residencial en la ciudad de Ibagué desde la década del treinta del siglo XX hasta el 2015, periodo que incluye cuando entra en vigencia el modelo económico neoliberal en el país con la política estatal de apertura económica. De esta forma, se determina a partir de las escalas de grado y de valor el rol desempeñado tanto por los agentes como por los actores urbanos en la configuración del territorio, un cambio del patrón tradicional de la segregación a una nueva tendencia de este fenómeno, expresado a través del proceso de ocupación y distribución de los grupos poblacionales conforme se fue dando la expansión urbana de Ibagué.

Palabras clave: Segregación. Ciudades intermedias. Espacio urbano.

\section{Resumo}

Considerando o caso específico da cidade de Ibagué, Colômbia, este artigo mostra uma análise geohistórica e contextual que apresenta os fatores determinantes da segregação residencial na cidade de Ibagué entre os anos 30 do século 20 e 2015, período que inclui a entra em vigência o modelo econômico neoliberal no país com a política estadual de abertura econômica. De este modo, determina-se a partir das escalas de grau e valor do papel desempenhado pelos agentes e atores urbanos na configuração do território, haja uma mudança do padrão tradicional de segregação para uma nova tendência desse fenômeno, expressa através do processo de ocupação e distribuição de grupos populacionais à medida que a expansão urbana de Ibagué ocorreu.

Palavras-chave: Segregação. Cidades intermediárias. Espaço urbano. 


\section{Abstract}

Considering the specific case of the city of Ibagué, Colombia, this article shows a geohistorical and contextual analysis that presents the determining factors of residential segregation in the city of Ibagué since the 1930s to 2015, period that includes when it comes into force the neoliberal economic model in the country with the state policy of economic opening. In this way, it is determined from the grade and value scales the role played by both agents and urban actors in the configuration of the territory, there is a change from the traditional pattern of segregation to a new trend of this phenomenon, expressed through the process of occupation and distribution of population groups as the urban expansion of Ibagué took place.

Keywords: Segregation. Intermediate cities. Urban space.

\section{Introducción}

Los efectos sociales, políticos, culturales y ambientales generados por el modelo de desarrollo urbano en función del modelo de acumulación capitalista (De Mattos, 2012), han hecho que la segregación residencial haya tomado relevancia en los estudios territoriales. Considerando que las ciudades no son territorios homogéneos (y por lo tanto su componente material y simbólico tampoco lo son), en las ciudades latinoamericanas la expresión de la segregación se ha transformado desde la introducción del neoliberalismo y ha puesto en evidencia la distribución diferencial de los grupos poblacionales, tanto a nivel macro como a micro escala (Sabatini, 2003).

En este sentido, el estudio de la segregación residencial en Colombia es relevante porque permite entender las nuevas expresiones de este fenómeno desde una escala de grado y por consiguiente nuevas dinámicas de la desigualdad socio-espacial e injusticia territorial desde una escala de valor. En este contexto se retoma el abordaje de la segregación residencial, fenómeno que da cuenta de la localización de las clases sociales por nuevas preferencias residenciales y la estratificación ${ }^{1}$ socioeconómica de los barrios que está en función de los precios del suelo, la precarización de la población e informalidad urbana, entre otros referentes de la estructura socioespacial de las ciudades.

Por otro lado, el fenómeno de la segregación residencial tiene una producción científica más desde el abordaje cuantitativo principalmente desde dos dimensiones: la concentración espacial de los grupos sociales y homogeneidad social de las diferentes áreas internas de las ciudades (Sabatini, Cáceres, \& Cerda, 2001). De este modo, es posible conocer el nivel de segregación residencial en las ciudades y deducir mecanismos de reproducción de las desigualdades sociales. Sin embargo, la dimensión subjetiva de la segregación que se refiere al prestigio y/o desprestigio de los diferentes barrios de la ciudad, ha sido poco abordada. Por tal razón, para contribuir a los estudios de la dimensión subjetiva de la segregación residencial, se consideró importante analizar, desde un contexto socio geo-histórico a este fenómeno y su relación con la teoría de la justica de Nancy Fraser.

Se considera que en Ibagué las acciones de los agentes, tanto privados como públicos, han transformado al territorio al ser los responsables en determinar hacia donde crece la ciudad y en especial donde se localizan los grupos poblacionales de acuerdo a su condición socioeconómica porque, el mercado restringe las posibilidades de elección de la vivienda. Con esto lo que han logrado es separar, segmentar y segregar a la población que habita en Ibagué, en lugar de plantear procesos

\footnotetext{
${ }^{1}$ La estratificación socioeconómica en Colombia es una política pública que tiene como fin otorgar subsidios a la población pobre (Uribe, 2008). Para lograrlo se clasifican a las viviendas de acuerdo a sus características y al entorno en el que se localizan, con el objetivo de dar una aproximación a la diferenciación socioeconómica jerarquizada. La categorización de las viviendas por estrato se definen así: estrato bajo (uno: bajo-bajo; dos: bajo y tres: medio-bajo), estrato medio (cuatro: medio) y estrato alto (cinco: medio-alto y seis: alto).
} 
que conlleven a la integración socioespacial. Por lo tanto, cabe preguntarse por cómo se manifestó la segregación residencial en el municipio de Ibagué desde la década de los treinta hasta el 2015.

El objetivo general de este documento es identificar las causas y consecuencias de las acciones de los diferentes agentes y actores urbanos, en la configuración social del territorio que conllevan la existencia de segregación residencial en Ibagué. Por lo tanto, se realiza una metodología históricoespacial desde una perspectiva diacrónica en la cual se establece a partir de la década de los años treinta hasta el 2015, cuáles han sido las acciones de los agentes en la organización espacial de Ibagué y cómo esta ha promovido la segregación residencial en la ciudad. Por lo tanto, a través de fuentes documentales de distintos autores (Clavijo, 2009; Espinosa, 1992; Francel, 2017; González, 2003, 2006; López, 2018) se construye el proceso de ocupación en Ibagué y con el apoyo de la cartografía oficial (Alcaldía de Ibagué, 2008; Secretaría de Planeación, 2014) se determina hacia donde se expande la ciudad y sus efectos en el territorio a partir de elementos socioespaciales y de desarrollo urbano.

De esta manera, es necesario explorar las implicaciones de la segregación residencial sin tomar juicios de valor, porque de lo que se trata es darle sentido al contexto geohistórico con análisis del discurso desde un territorio específico (Saraví, 2008), como lo es Ibagué. En este sentido, el concepto de segregación residencial aquí se entiende desde la definición más genérica es decir, como la distribución espacial de los grupos poblaciones que internamente son homogéneos por características sociales, económicas y espaciales pero diferentes entre grupos. No obstante, al reconocer que este fenómeno ha cambiado su expresión con el paso del tiempo se considera pertinente abordar en este artículo a la segregación desde la dialéctica geohistórica y espacial porque, a través de un análisis discursivo de modelos de ciudad (Borsdorf, 2003) manifiesta que la condición socioeconómica de la población se corresponde con el sector en el que habitan debido a las políticas de desarrollo urbano.

Este artículo está compuesto en cuatro partes: En primer lugar, se plantea una contextualización teórica de la segregación a través de los diferentes paradigmas que la han estudiado. En segundo lugar, se determina el cambio de la expresión de la segregación socioespacial. En tercer lugar, se presenta una caracterización socio geo-histórica de la segregación en la ciudad de Ibagué a partir de la década de los treinta del siglo XX hasta la década de los noventa y por último se describen las consideraciones finales.

\section{Segregación socioespacial en un contexto contemporáneo}

La segregación socioespacial es una categoría que ha tenido diferentes interpretaciones según la disciplina desde la que se estudie. Se encuentra que desde la sociología se interpreta como la ausencia de la interacción entre grupos sociales y desde la geografía se define como la desigualdad de los grupos sociales en un espacio físico (White, 1983). Sin embargo, esto no tiene una implicancia directa entre las dos concepciones porque, la existencia de una no necesariamente significa que la otra exista. No obstante, en lo que si se encuentra un consenso en la definición contemporánea de la segregación es que es consecuencia del desigual desarrollo urbano que presentan las ciudades latinoamericanas (Schteingart, 2001), por ello este fenómeno se conoce como "la aglomeración en el espacio de familias de una misma condición social, más allá de cómo definamos las diferencias sociales” (Sabatini, 2003, p. 7).

Este desigual desarrollo urbano ha causado que la expresión de la segregación cambie con el paso del tiempo, manteniendo una base de población pobre y de manera dispersa, en medio de ella, se encuentran unos fragmentos de riqueza. En primera instancia, se encuentra una expresión tradicional del fenómeno en un modelo de ciudad compacta (Bähr \& Borsdorf, 2005), que se caracterizaba por dividir socioespacialmente a los grupos poblacionales según su condición socioeconómica (Janoschka, 2002), lo que conllevaba a que la distancia geográfica desde el centro de la ciudad indicara la posición social de los sujetos, esto es, entre más cerca se localizara la vivienda del centro de la ciudad se consideraba que las personas que la habitaban contaban con un nivel socioeconómico alto, contrario a si se encontraban hacia la periferia se les consideraban de condición socioeconómica baja, por ello la 
densidad poblacional era más en la periferia que en las zonas cercanas al centro, que además contaban con mejores equipamientos colectivos .

Sin embargo con el cambio de patrón segregativo a uno nuevo, causado por una economía global que transforma al espacio en fragmentos de riqueza (Borsdorf, Hidalgo, \& Vidal-Koppmann, 2016), ya no es la distancia geográfica la que determina la segregación, lo hace la distancia social en un contexto urbano donde la mezcla y cercanía geográfica de los grupos poblacionales se hace cada vez más presente bajo el modelo de ciudad fragmentario (Bähr y Borsdorf, 2005). En este sentido, se mantiene la división socioespacial de los grupos poblacionales según su nivel socioeconómico y la densidad poblacional sigue siendo más alta en zonas geográficas con personas con condiciones socioeconómicas bajas.

Por lo anterior las ciudades latinoamericanas al presentar un desigual desarrollo urbano (Schteingart, 2001), han generado cambios en la expresión de la segregación socioespacial con el paso del tiempo. Antes de la década de los noventa en Latinoamérica la segregación se definía desde la distancia es decir, la posición social de la población se daba por la distancia física de su casa y el centro fundacional de las ciudades; cerca del centro se encontraba la clase social alta, en el anillo siguiente la población de condición social media y hacia la periferia la población de nivel social bajo. No obstante, la distancia en un contexto contemporáneo no necesariamente es una manifestación de segregación, porque si bien es cierto los grupos poblacionales disímiles están cada vez más cerca, esto no significa que haya integración socioespacial, por lo tanto, de acuerdo con el contexto en el que se estudie la segregación, se puede indicar que es un fenómeno complejo de analizar según las particularidades en las que las ciudades hayan evolucionado.

Por lo anterior, la segregación residencial es un fenómeno que a través del tiempo ha presentado dos expresiones como se puede observar en la tabla 1. La primera se conoce como patrón tradicional de la segregación y fue desarrollada en gran parte del siglo XX, tiene como principales características la alta concentración espacial de los grupos socioeconómicos altos y con tendencia a expandirse en forma de cono hacia la primera periferia; localización de la población socioeconómicamente vulnerable en las zonas periféricas de las ciudades, las cuales se destacan por tener equipamientos colectivo precarios, como también ubicaciones en el centro deteriorado de la ciudad; posible diversidad social en los barrios con sujetos de condición socioeconómica alta; conformación de la ciudad dual; y estructura a gran escala² de la segregación. La segunda es la nueva expresión de la segregación, desarrollada primordialmente con las reformas económicas y urbanas bajo el modelo de liberalización de los mercados del suelo (Sabatini y Cáceres, 2005), contrario al patrón anterior, el nuevo se caracteriza por tener una expresión de la segregación a pequeña escala geográfica; se rompe con la alta concentración de las élites; construcción de multicentrismo comerciales, de oficinas y de servicios; el alza generalizada de los precios del suelo y por ende su escasez; expansión residencial de los grupos de élite en la zona periférica de la ciudad en condominios o vivienda tipo campestre; y procesos de renovación urbana.

\footnotetext{
2 La escala en este trabajo es entendida como como la magnitud que tiene la superficie geográfica en la que cual se expresa un fenómeno es decir, no se refiere a la escala de un mapa. Para Francisco Sabatini (2003) la segregación a gran escala la representa la concentración espacial de los grupos poblacionales de condición socioeconómica media y alta en el vértice del centro histórico de la ciudad y a las amplias áreas de la periferia ocupada por la población de nivel socioeconómico opuesto.
} 
Tabla 1 - Características de los cambios de patrones de la segregación residencial en Latinoamérica

\begin{tabular}{|c|c|c|}
\hline Ítem & Expresión tradicional de la segregación & Nuevo patrón de la segregación \\
\hline 1 & $\begin{array}{l}\text { Concentración de la población de ingresos altos } \\
\text { alrededor del centro histórico de la ciudad, } \\
\text { conocido como cono de alta renta. }\end{array}$ & $\begin{array}{l}\text { Dispersión de las élites fuera del cono de alta } \\
\text { renta, generando una expresión a menor } \\
\text { escala de la segregación. }\end{array}$ \\
\hline 2 & $\begin{array}{l}\text { Agrupación de población de ingresos bajos en las } \\
\text { zonas periféricas de la ciudad y en zonas } \\
\text { deterioradas del centro de la misma. }\end{array}$ & $\begin{array}{l}\text { Surgimiento de subcentros comerciales, locales } \\
\text { comerciales y de servicios fuera del Centro y en } \\
\text { general de los barrios de población de ingresos } \\
\text { altos, con el fin de conseguir extensas áreas de } \\
\text { mercado. }\end{array}$ \\
\hline 3 & $\begin{array}{l}\text { Diversidad relevante de grupos sociales de ingresos } \\
\text { medios en el cono de alta renta. }\end{array}$ & $\begin{array}{l}\text { Tendencia al alza de los precios del suelo y su } \\
\text { entorno espacial urbano, generando una } \\
\text { propensión de la localización de viviendas } \\
\text { nuevas para los grupos poblacionales de } \\
\text { ingresos bajos en la periferia de la ciudad o } \\
\text { zonas aledañas. }\end{array}$ \\
\hline 4 & $\begin{array}{l}\text { Conformación de una posible ciudad "dual", en la } \\
\text { que por un lado se tiende a estigmatizar aquellas } \\
\text { zonas sin prestigio de la ciudad y, por el otro, a dar } \\
\text { importancia solo a zonas modernas de la ciudad. }\end{array}$ & $\begin{array}{l}\text { Surgimiento de formas de crecimiento } \\
\text { residencial discontiguas respecto de la ciudad, } \\
\text { tanto en favor de centros urbanos menores } \\
\text { como de la ocupación rural. }\end{array}$ \\
\hline 5 & $\begin{array}{l}\text { Abordaje de la segregación a gran escala } \\
\text { geográfica, lo que ha forzado la imagen de ciudad } \\
\text { "dual" a pesar de la diversidad de grupos sociales } \\
\text { de ingresos medios en el cono de alta renta. }\end{array}$ & $\begin{array}{l}\text { Procesos de renovación urbana de áreas } \\
\text { centrales, procurando por aquellas casas } \\
\text { antiguas deterioradas con las que se logre de } \\
\text { nuevo un uso residencial o uno nuevo terciario. }\end{array}$ \\
\hline
\end{tabular}

Fuente: Con base a Sabatini (2003) y Sabatini y Cáceres (2005).

En este sentido, es relevante indicar que los cambios que se han dado en la expresión de la segregación han transformado la perspectiva de grado (distancia, división y distancia) de este fenómeno ya que la posición social de los sujetos (López, 2018) estaba determinada en el patrón tradicional de la segregación por la distancia de acuerdo a la ubicación de la plaza central (Bähr y Borsdorf, 2005). No obstante, en la nueva expresión de este fenómeno lo que se encuentra es que las escalas de grado cambian su forma, es decir, la posición social ya no es determinada por la posición geográfica; la densidad poblacional es baja pero el consumo del suelo es creciente, se han desarrollado nuevas lógicas de separación a través de las fronteras que permiten aglomerar a los grupos sociales de acuerdo a atributos en común y a su nivel social.

Sin embargo, la construcción de proyectos inmobiliarios en conjuntos cerrados no es propia de aquellos sujetos a los que les interesa mantener un prestigio social, ostentar su riqueza, dar una impresión de privilegio y de lujo; también los hay para los grupos de condición socioeconómica baja. En lo que se diferencian estos proyectos más allá del encerramiento, es la diferenciación de sus equipamientos privados y la calidad del servicio de seguridad que se les ofrece. No obstante, para ellos vivir en urbanizaciones cerradas "no es tan sólo cuestión defensiva, sino también un elemento de distinción económica, de expresión de su poder y de su diferencia, así como el deseo de reafirmar el valor financiero de sus propiedades" (Cortés, 2010, p. 125).

De esta manera se tiene que la segregación residencial, analizada además como el acceso desigual a los bienes materiales y simbólicos de la ciudad, es relevante, procurando una perspectiva de "espacialización de la estratificación de la sociedad y, (...) comprendida como consecuencia de lógicas colectivas cuyo fundamento son las relaciones sociales" (De Queiroz, 2003, p. 41).

La segregación residencial es causada principalmente por la acción del Estado, el mercado inmobiliario y la población en relación con el mercado de trabajo (Torres, Marques, Ferreira, \& Bitar, 
2003). El Estado incide en la disminución o aumento de la segregación de acuerdo a las normas habitacionales que postule $\mathrm{y}$, por lo tanto, tiene la capacidad de distribuir a los grupos poblacionales en un lugar y no en otro, promoviendo o no la integración social. No obstante, se encuentra que el Estado ha profundizado las distancias tanto físicas como sociales de la población (Hidalgo, 2007). Por su parte y con la complacencia del Estado, el mercado inmobiliario ha cambiado la estructura urbana a través de las transformaciones económicas y sociales generadas por un nuevo modelo de ciudad bajo un Estado neoliberal, que ha permitido la producción de artefactos urbanos como centros comerciales, urbanizaciones privadas y parques empresariales, entre otros (Ciccolella \& Vecslir, 2010).

Por otro lado, al tener un acceso desigual al suelo como producto del mercado de trabajo flexible, la población se enfrenta a una coexistencia con grupos poblacionales con diferencias socioeconómicas entre sí y características homogéneas al interior de ellos. Estas causas tienen su punto de encuentro cuando el Estado - que tiene como función promover la demanda de vivienda a través de subsidios, y el promotor inmobiliario- hacen uso del subsidio como mecanismo de atracción de nuevas demandas residenciales (Contreras, 2017).

En consecuencia, a partir de la transformación urbana que han tenido las ciudades Latinoamericanas a causa de la política neoliberal reflejada en la producción social del territorio en función de los modelos económicos, es pertinente indicar las características principales de las escalas de valor que puede llegar a tener la segregación socioespacial (redistribución, reconocimiento y representación) y que abordan a la justicia desde una postura multidimensional. Justicia que es importante retomar cuando el neoliberalismo con su ideología de privatización ha profundizado las diferencias socioeconómicas de las personas según su posición social y posesión de capital.

Para empezar, a causa de los procesos de globalización y transformación económica con efectos locales en dimensiones como la economía, la política y la cultura, se dan dos injusticias, por un lado, la mala redistribución socioeconómica teniendo como causa principal la producción capitalista a escala transnacional que busca disminuir costos en la cadena de valor de los bienes o servicios, principalmente en las condiciones laborales y que las encuentran en países en vía de desarrollo con estructuras económicas que no brindan ingresos necesarios para que toda la población pueda participar de manera equitativa en sociedad, por ello se considera que la riqueza se redistribuya y que con el paso del tiempo no se les siga negando los recursos que necesitan para interactuar con los demás como pares (Fraser, 2008a). Esta escala de valor se relaciona con una de las consecuencias de la segregación y es desigualdad socioespacial que se evidencia en la nueva expresión de este fenómeno bajo un modelo de ciudad fragmentario.

Por otra parte, se encuentra la injustica desde el reconocimiento fallido o la desigualdad del estatus, la cual no es propiamente una consecuencia de la mala redistribución poblacional, se considera que estas injusticias tienen una relación bilateral porque los grupos poblacionales excluidos demandan por parte del Estado justicia social frente a situaciones que los dejan en condición de desposeídos social y económicamente, situaciones que han "estigmatizado el rol de las expresiones culturales de quienes se dice, son indignos y no merecedores de habitar la ciudad" (Lacarrieu, 2007, p. 50). No obstante, esta justicia social se debe reclamar desde el respeto a la diversidad como solución permanente al problema de injustica social. Por ello la filósofa Nancy Fraser (2008b) plantea que se debe de velar por la paridad participativa de los sujetos, sin esto lo que va a seguir permaneciendo es la mala redistribución y el mal reconocimiento. Esta escala de valor se relaciona con otra de las consecuencias de la segregación que es la dualización de la ciudad, en el que de acuerdo al estatus de la población se estigmatiza a la población por su condición socioeconómica baja, mientras que el grupo opuesto goza de prestigio social por la localización moderna de sus viviendas.

La tercer injusticia se da desde la representación fallida la cual hace énfasis en que "los límites políticos y/o las reglas de decisión funcionan injustamente negando a determinadas personas la posibilidad de participar en paridad con otras en la interacción social -incluida la que se da en el 
terreno político aunque no sólo en éste" (Fraser, 2008a, p. 43). Por ello Fraser (2008a) considera necesario pasar de una representación fallida a una plena, donde no sólo haya alguien que represente a los grupos poblacionales desposeídos sino que ellos mismos se puedan representar en el ámbito político. Además, hay otro problema político que es el del desenmarque, lo que significa que buscar representación política en el Estado nación no es suficiente cuando el Estado deja expuesto a estos sujetos a las arbitrariedades que vienen más allá de las fronteras, en el marco de una globalización excluyente promovida por la dinámica de las empresas e instituciones transnacionales y de telecomunicaciones en un contexto de ciudades globales (Méndez, 2001; Sassen, 2015). Por último, esta escala de valor se puede relacionar con una de las consecuencia de la segregación que es la tendencia al alza de los precios del suelo y su entorno espacial urbano, el cual genera una propensión de la localización de viviendas nuevas para los grupos poblacionales de ingresos bajos en la periferia de la ciudad o zonas aledañas, lo que genera un juego de inclusión y exclusión, se incluye en mejores zonas y mejor dotadas a las personas con el capital suficiente para pagar por estos servicios y se excluye y segrega a la población que no tiene otra alternativa que aceptar lo que el mercado inmobiliario le ofrezca en áreas de menor calidad.

Finalmente y por todo lo anterior, es preciso exponer desde una perspectiva contextual y geohistórica, cómo desde la década de los treinta del siglo XX Ibagué ha tenido una tendencia del desarrollo urbano en función del crecimiento planeado y espontáneo, y los proyectos de ciudad regida por las directrices del urbanismo desde la época colonial.

\section{El caso de Ibagué, Colombia}

Ibagué en la primera etapa de la urbanización durante el periodo colonial contaba con el trazado en damero que empezaba en el centro en su plaza fundacional y que proyectaba a expandirse hacia la zona nororiental, pues en la zona noroccidental la montaña la rodea como límite físico al núcleo urbano (Figura 1). En la década de los cuarenta del siglo XX se gestó un crecimiento lineal desde la zona céntrica, que reconfiguró territorialmente a la ciudad en torno a las vías de interconexión nacional y regional para integrar a la ciudad a la economía del país bajo el proyecto de modernización liderado por los gobiernos liberales de la época, por lo tanto se dieron adecuaciones en la industria, principalmente del arroz, el algodón y el sorgo, interconexión que ha sido característica de la ciudad desde su fundación hasta el presente, porque ha cumplido con el papel de centro de enlace con otros municipios que se iban tejiendo en una red de carácter regional y de vinculación con Santafé de Bogotá (Rivera, 2016).

En este contexto incipiente de modernización, el desarrollo urbano de Ibagué siguió las tendencias del urbanismo de la época y planeó el Plan Urbanístico Futuro (1935), el cual tenía ideas higienistas y de ciudad jardín que ya tenían aplicaciones en Europa, Norteamérica y Latinoamérica. Esto se explica en tanto que, en la primera mitad del siglo XX, en el centro se proyectó la construcción de viviendas tipo "casa quinta" para la clase media alta y alta de la ciudad; paralela a esta situación, la clase media y baja se localizaba en las zonas de ladera y hacia la periferia de la ciudad, conservando de esta manera una segregación residencial a gran escala (Sabatini, 2003).

Como muestra de la inscripción de la ciudad a las dinámicas nacionales se encuentra que el desarrollo de las ciudades colombianas a partir de la década de los años treinta del siglo XX estaba guiado por cánones internacionales que tenían como objetivo establecer criterios que no permitieran "la formación de barrios de excesiva concentración, faltos de aireación y de luz y acordar la presencia de áreas verdes privadas y comunes para mejorar las condiciones de vida y la estética urbana” (Torres et al., 2009, p. 234). 


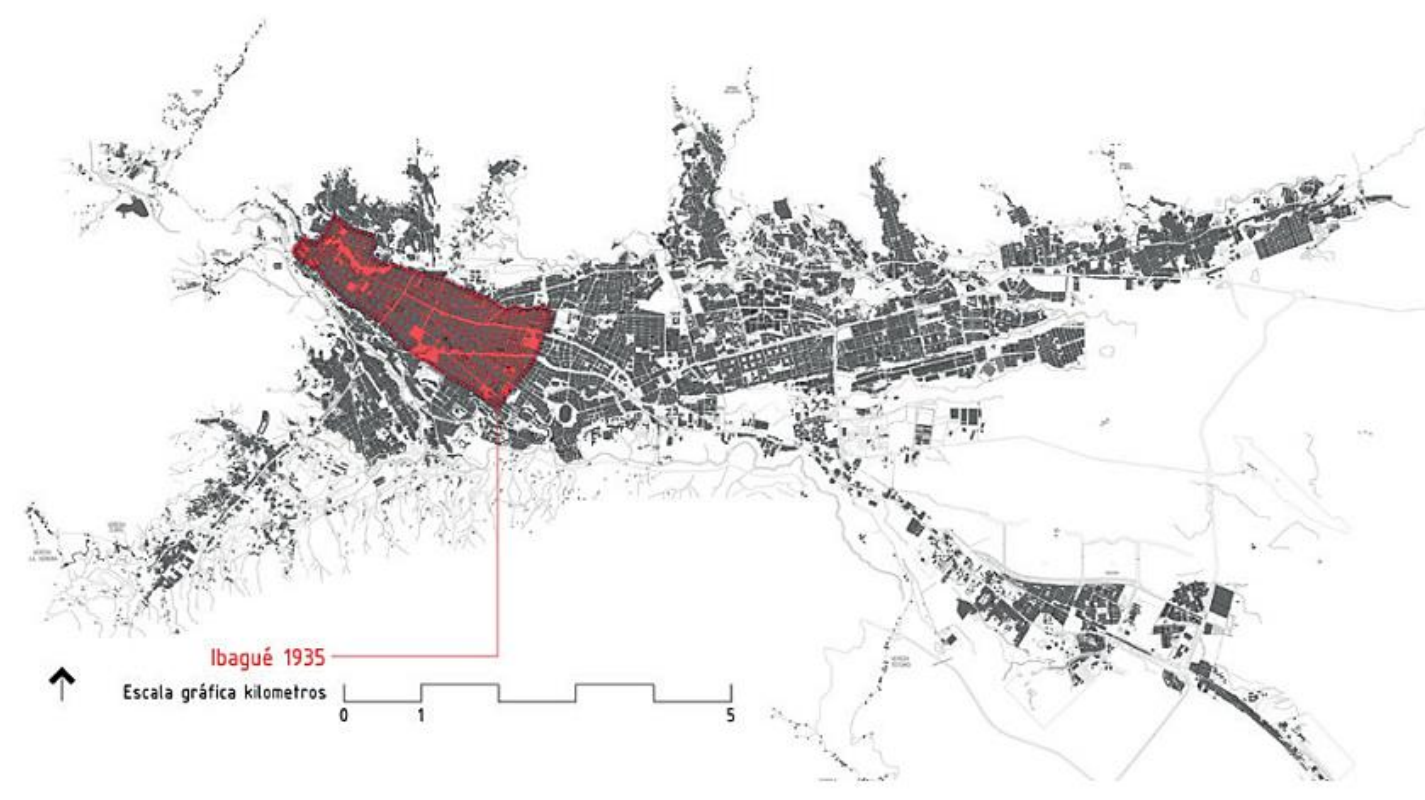

Figura 1 - Extensión de lbagué en 1935 con respecto al plano actual Fuente: Francel (2017, p. 24).

Lo que evidencian estos enfoques es la historia de las mentalidades urbanas lideradas principalmente por la élite local, porque expresan nuevas expresiones de segregación residencial y distribución espacial de los diferentes grupos sociales de acuerdo a su condición socioeconómica y de su inserción en sectores productivos emergentes, así como también la consolidación de una burocracia local.

Además, desde la década de los treinta, periodo de avance institucional, el Instituto de Crédito Territorial (ICT) y el Banco Central Hipotecario (BCH) como entidades del Estado estaban encargadas de la producción de vivienda obrera especialmente para empleados, es decir la clase media, por ello generan un tipo de vivienda diferente al rural y es ahí donde aparecen los barrios obreros y las casas en serie financiadas. A partir de los años cuarenta, la población de condición socioeconómica alta empieza a abandonar el centro de la ciudad y se va ubicando en la periferia y construyendo viviendas urbanas de muy buena calidad (González, 2003), ejemplo de ello es el barrio Interlaken y posteriormente el barrio Cádiz, Piedra Pintada, El Vergel y otros (Figura 2).

En efecto, la segregación residencial en la ciudad tuvo hasta el cuarto decenio del siglo XX una expresión espacial propia del periodo de modernización, la cual correspondió a la estructura urbana sectorial y que se prolongó hasta la década de los noventa con una estructura de cono de privilegio de la clase alta y el inicio del proceso de suburbanización. Por el contrario, la clase media y baja que se localizaba hacia la periferia marginal de la ciudad estableciendo así una segregación residencial a gran escala propia de ciudades latinoamericanas (Sabatini, 2003).

Por lo tanto, lo que se evidencia desde el análisis geohistórico, es que hasta la década de los años cincuenta del siglo XX, la polarización entre barrios de clase alta y de desarrollo espontáneo, se originan a partir del mantenimiento de unos privilegios económicos y políticos de la tradicional élite latifundista del Tolima, y de otra parte, de la localización involuntaria de la población con condición socioeconómica baja cerca a los afluentes hídricos y zonas de ladera expuestas a riesgos. En este sentido, un ejemplo de esta dinámica es la comuna seis, que se ha caracterizado por construir asentamientos informales, que según lo plantea el arquitecto Carlos Torres y otros autores (2009), barrios como San Antonio, Gaviota y las Delicias, se conocen por ser receptores de población desplazada por el conflicto armado. 


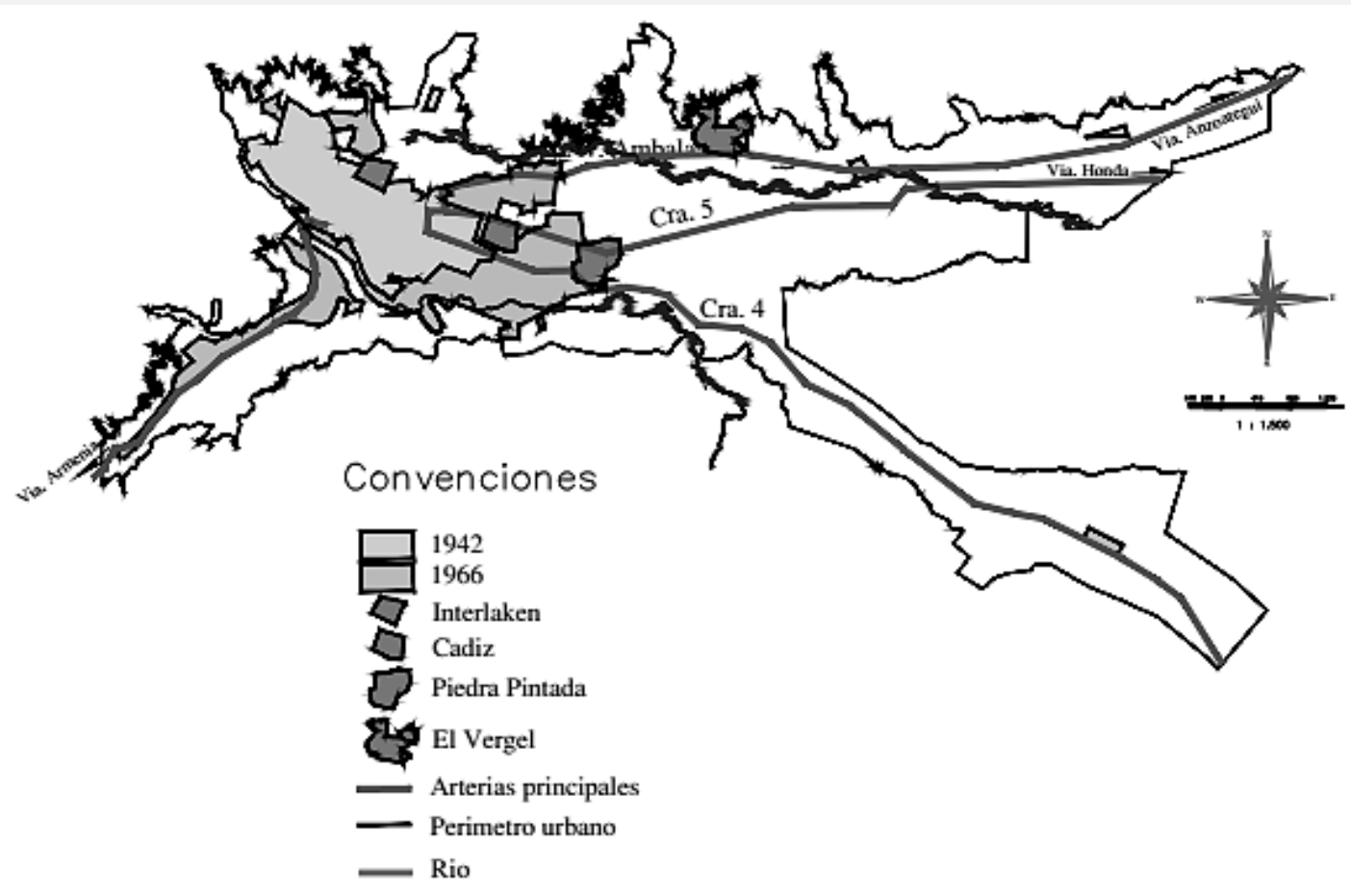

Figura 2 - Proyectos inmobiliarios en la periferia de la ciudad, años cuarenta del siglo XX. Fuente: González (2003) y Espinosa (1992).

Como consecuencia, el proceso de expansión en Ibagué se generó de manera paralela a la violencia política de la época y el desplazamiento forzado de la población rural hacia la ciudad, éxodo que propició un incremento poblacional: la población se duplicó en el periodo comprendido entre 1938 y 1951 ya que pasó de 27448 a 54347, respectivamente (Espinosa, 1992).

La situación de crecimiento de población generó un impacto espacial en la ciudad, expresado entre el tramo oriente y occidente; en las inmediaciones de los ejes viales de la carrera cuarta (Avenida Ferrocarril), quinta y la catorce (Avenida Ambalá), se encontraban barrios dispersos entre legales y no legales, y entre ellos lotes vacíos que para la época se denominaban lotes de engorde, morfología precedida por el clientelismo político del momento, interesado en las rentas urbanas. Un ejemplo de ello fue la construcción del barrio Belén (ver Figura 3), beneficiado por la construcción vial del centro -propuesta desde el Concejo Municipal-y de equipamientos como el Parque centenario y el Colegio Tolimense, contribuyendo a la valorización del suelo, mientras que hacia afuera se encontraban los barrios precarios (González, 2006).

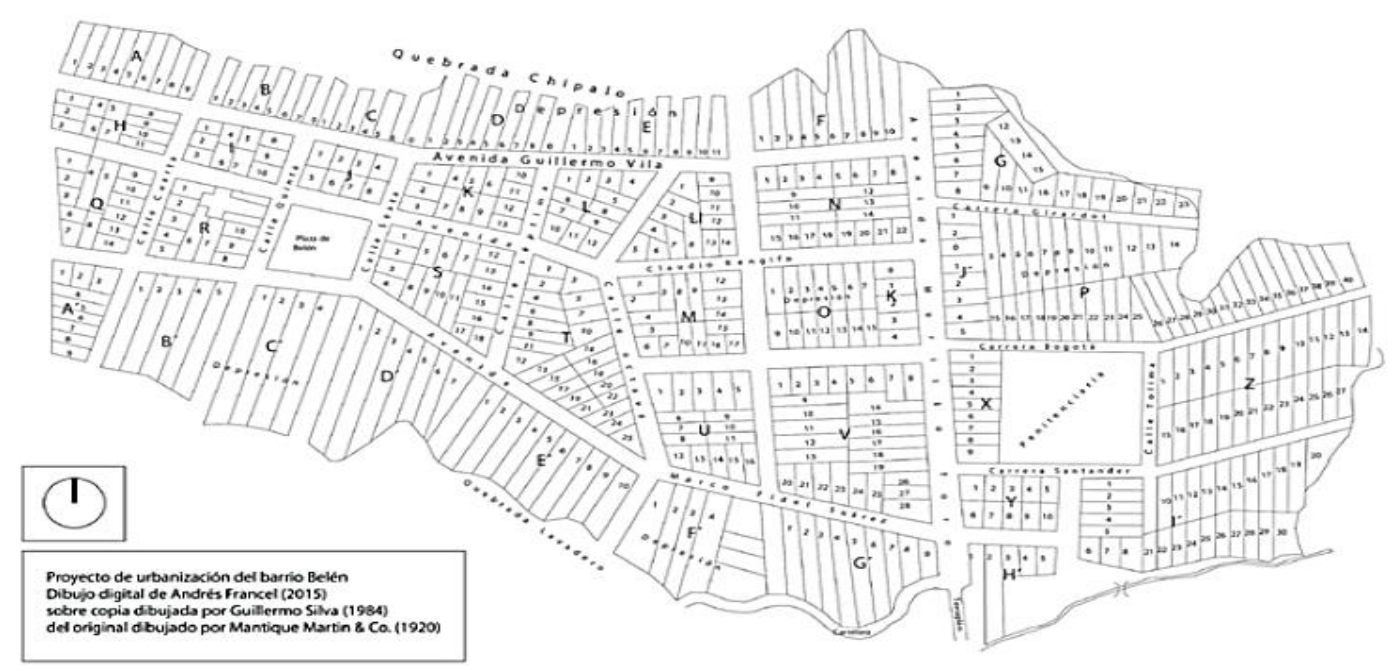

Figura 3 - Plano del proyecto de construcción del Barrio. Fuente: Francel (2017, p. 30). 
En síntesis, desde la primera mitad del siglo XX hasta la década de los setenta se acentuaron dos patrones paralelos de expansión, es decir, una dualización de la ciudad (Prévôt-Schapira, 2000). Por un lado, en el mercado convencional se otorgaban casas a través del Estado; por otra parte, en el mercado no convencional se promueve la vivienda autoconstruida por la población con déficit habitacional y que no cuenta con los recursos necesarios para poder adquirirla y que se ve impulsada a buscar una ocupación forzada del suelo, expresada en formas no legales de construcción de vivienda y de servicios colectivos básicos. No obstante, la población de nivel socioeconómico bajo que no contó con la oportunidad de adquirir su casa en barrios debidamente planificados, lo que deja en evidencia es la ausencia de representación a partir de las políticas urbanas de vivienda, abastecimiento de servicios públicos y equipamiento. De esta manera se expresa el fenómeno de la segregación residencial, promovida desde el gobierno local y con fines económicos de los terratenientes urbanos de la época.

Así mismo, la población inmigrante fue construyendo su propia ciudad de manera espontánea; a su vez, la improvisación del gobierno local contribuyó a que la ciudad se expandiera sin previa planificación. De esta manera, se fomentó una escasa y dispersa reglamentación de desarrollo de ciudad, en la que todos pudieran disfrutar de las inversiones en infraestructura urbana por parte de la Alcaldía de turno. La zona de expansión de la ciudad se fue dando sin continuidad entre barrios y en función de las vías; desde aquella época, esta dinámica determinó una tendencia de localización dispersa entre barrios de diferentes clases sociales.

Aunque la población inmigrante no tenía en Ibagué una participación significativa en la década de los setenta y ochenta -como la tuvo a mediados del siglo XX-, siguió siendo un determinante de la expansión urbana y en la necesidad de considerar usos del suelo urbano. Así mismo, un nuevo desplazamiento coyuntural por la avalancha de Armero a finales de la década de los años ochenta, contribuyó a que se plantearan políticas económicas de corto y largo plazo para mitigar las necesidades de la población que quedó vulnerable y atraer capitales para reactivar la economía en una ciudad que adquirió una dependencia principalmente de ciudades como Bogotá.

Esta reactivación económica ibaguereña influyó en la zona urbana (Figura 4). Entre la avenida Mirolindo y el Barrio Picaleña (suroriente) se estableció la industrialización y las soluciones habitacionales para los desplazados por la avalancha; la tendencia de construcción continuó en la zona nororiental y de manera paralela se presentó la autoconstrucción de vivienda precaria alrededor del Río Chipalo, los sectores del Salado (nororiente), Ambalá (norte) y Picaleña (suroriente), entre otros (Figura 5). Sin embargo, la ciudad no tenía una expansión urbana adecuada en función del crecimiento de las actividades comerciales e industriales y se vio afectada de nuevo por el déficit habitacional con este desplazamiento. 


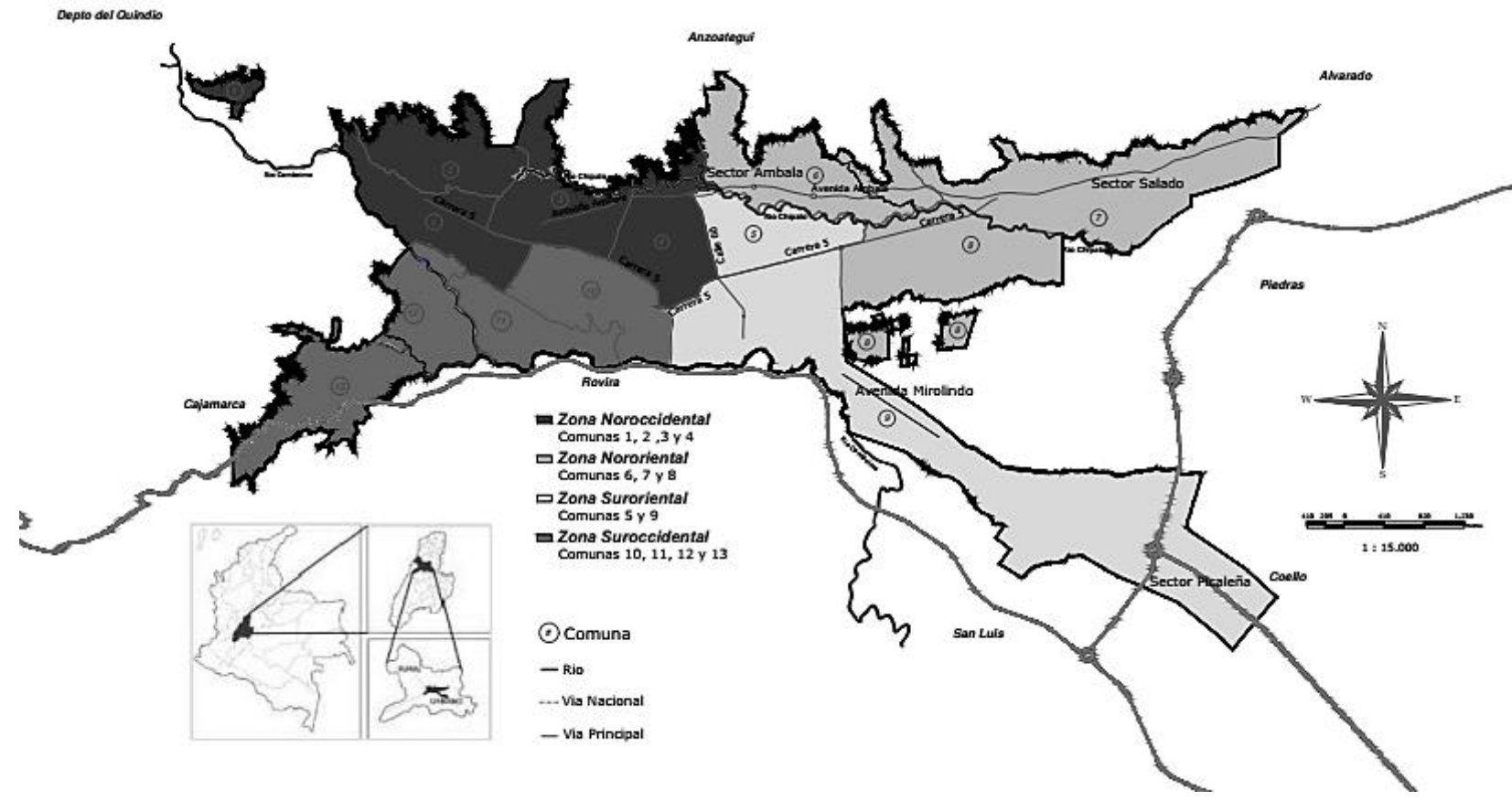

Figura 4 - Zonas de reactivación económica de lbagué. Fuente: Torres et al., (2009) y Alcaldía de lbagué (2008).

Lo que se puede evidenciar en este periodo es que hay un reconocimiento fallido porque se niega la posibilidad a un grupo poblacional en específico de estar en igualdad de condiciones para adquirir vivienda, por lo tanto el estatus con el paso del tiempo se consolida en el grupo de personas con nivel socioeconómico medio y alto, lo que hace que se desvalorice a la persona de condición socioeconómica baja y esto provoca separación, exclusión y segregación (Fraser, 2008b). Esto sucede porque existe una generalización al estigmatizar a la población de bajos ingresos como consecuencia de la capacidad que tiene el mercado de segregar el espacio cuando se apropia materialmente de los bienes escasos (públicos o privados) que se distribuyen en él según el capital que poseen sus demandantes potenciales y "éste permite mantener a distancia a las personas y las cosas indeseables, al mismo tiempo que acercarse a las personas y las cosas deseables (Bourdieu, 1999, p. 122).

Por otro lado, a partir de la década de los noventa del siglo XX, con el abandono del control y la gestión del Estado en el sector de la vivienda, hábitat y territorio, se dio paso a la apropiación privada de las formas urbanas por parte de los actores privados, así, se implementó a nivel nacional la denominada fase del sistema de Vivienda de Interés Social (VIS) ${ }^{3}$, que inició bajo la premisa de la ineficiencia del Estado en la producción de vivienda para afrontar el déficit habitacional y la necesidad de descentralizar los recursos para que los municipios pudieran intervenir en este proceso. De esta manera, el Estado tomaba el rol de incentivar la demanda de la vivienda y la oferta quedaba en manos del mercado inmobiliario (Ceballos, Saldarriaga, \& Tarchópulos, 2008).

En Ibagué, las nuevas zonas urbanizadas bajo la modalidad de subsidio se fueron presentando en el suroriente y nororiente, en dirección a las vías carrera quinta y cuarta y en los sectores del Salado y Picaleña, lejos del centro tradicional entre 1998 y el 2014 (Figura 5). Las nuevas construcciones no se ubicaron de forma homogénea; en el extremo oriente y el nororiente se construyeron proyectos VIS y en el norte las NO VIS principalmente, a pesar de ser una zona de diversidad de estratos con predominancia en el 1 y 2 . Además, las áreas que representan esta estratificación permanecen en el

\footnotetext{
${ }^{3}$ Las VIS son proyectos de vivienda que tiene el Estado colombiano hacia la población de bajos recursos. Estas VIS tienen un valor equivalente entre 70 y 135 salario mínimo legal vigente (SMLV, por ello para que una persona pueda acceder a una vivienda de este tipo sus ingresos mensuales no deben superar los cuatro SMLV. Por su parte, las viviendas NO VIS, son proyectos inmobiliarios enfocados a la población que condición socioeconómica alta.
} 
tiempo con esta misma condición, por ejemplo en la zona suroccidental de la ciudad el estrato que predomina es el dos y es allí donde se construyeron proyectos de vivienda de interés prioritario (VIP) como el Tejar cerca de barrios precarios (Figura 6), es decir, "se ha dirigido la inversión en obras de asistencia barrial hacia áreas urbanas no convencionales, localizadas a mayor distancia del centro de la ciudad y donde el precio del suelo es menor" (Fernández de Córdova, 1994, p. 219).

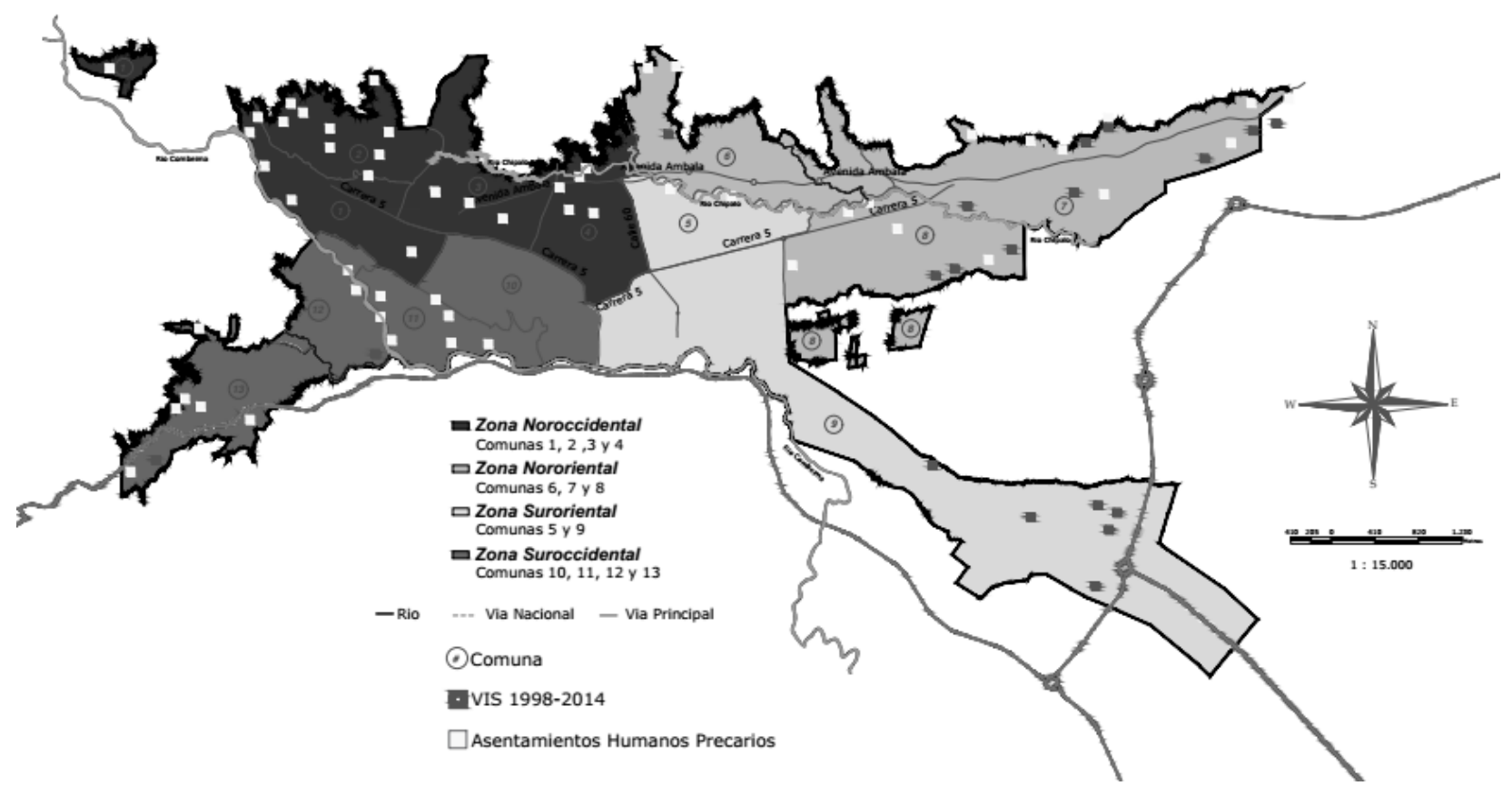

Figura 5 - Localización de viviendas VIS (1998-2014) y de barrio autoconstruidos de origen informal, Ibagué. Fuente: Elaboración propia en base a Clavijo (2009); Alcaldía de Ibagué (2008) y Secretaría de Planeación (2014).
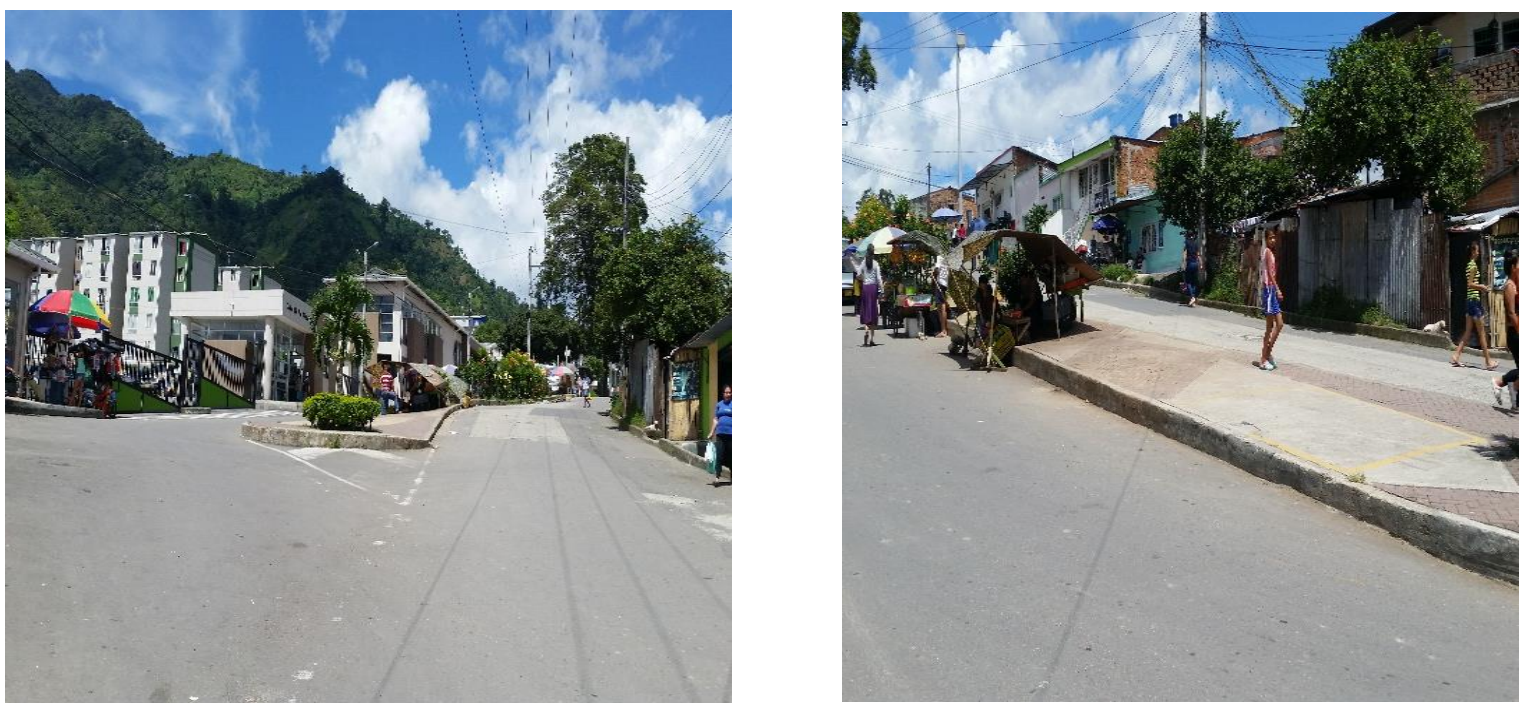

Figura 6 - Comuna 13, Ibagué. Zona urbana. Conjuntos cerrados de VIP -El Tejar, izquierda- vs Casas tradicionales precarizadas, Boquerón. Fuente: Fotografías de la autora, Mayo 2017

Por lo anterior, se encuentra una falta de redistribución que profundiza la división socioespacial de los grupos poblacionales y que al estar basada en una estructura socioeconómica capitalista de la sociedad, es un obstáculo para la paridad participativa (Fraser, 2008b). Esto se debe a que son las desigualdades económicas las que impiden participar a las personas en igualdad de condiciones por un mejor techo, frente aquellos que tiene mejor nivel socioeconómico. Esto no quiere decir que la oferta 
de vivienda se deba dar solo en función de una mezcla de barrios con diferenciación en su estratificación, porque como se puede evidenciar en la ciudad de Ibagué en el norte existe diversidad de estratificación socioeconómica que no ha tenido efectos positivos de la segregación, por ello pensar en la posibilidad de movilizar los recursos a las personas y no movilizar las personas hacia los recursos (Ruiz-Tagle \& López, 2014), podría ser una opción de transformación al sesgo neoliberal.

Por otro lado, a partir de la primera década del siglo XXI en el centro geográfico de la ciudad se construyeron centros comerciales (Multicentro, La Estación y Aqua Power Center) sobre la calle 60 (figura 7), conocida como la milla de oro por el boom inmobiliario comercial y de vivienda que se ha gestado en esa área y que pasó de ser estrato medio a estrato medio alto, lo que generó una nueva división espacial de cultura y de consumo; así, las actividades comerciales que se gestaban en el centro tradicional de la ciudad se descentralizaron y surgieron nuevos subcentros comerciales.

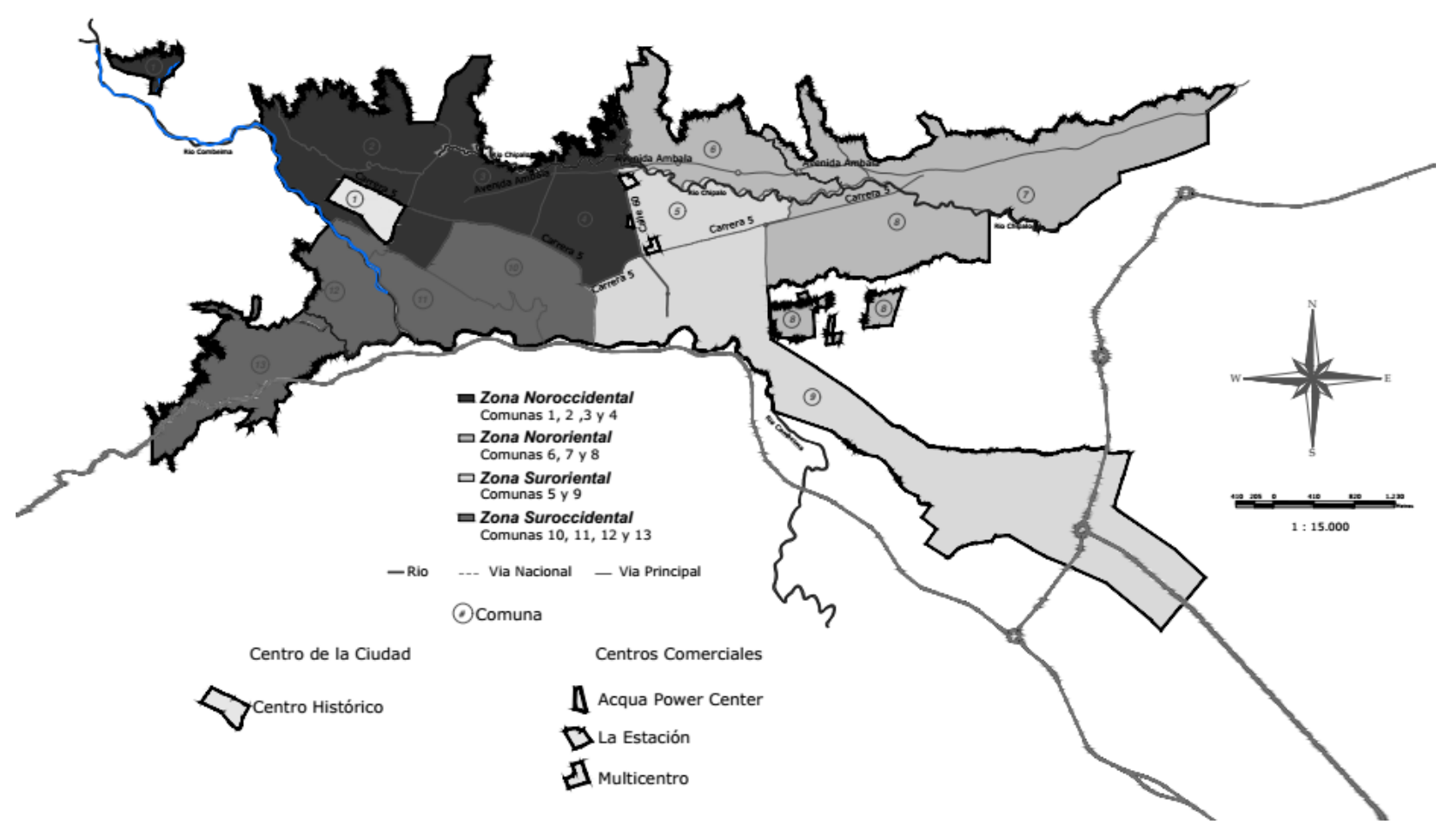

Figura 7 - Centro comerciales La estación, Multicentro y Aqua Power Center. Fuente: Elaboración propia con base en Secretaría de Planeación (2014).

De esta manera se fomentó que el proceso de expansión residencial de la ciudad quedara en manos de las leyes del mercado. Además, la entrada en vigor del nuevo modelo neoliberal implementado en el caso colombiano a partir de la década de los noventa y la política de liberalización del mercado del uso del suelo, entre otros factores, contribuyó a un cambio en el patrón de la segregación residencial, no solo de las ciudades-región de Latinoamérica, sino de ciudades intermedias como Ibagué. Aunque para el caso de esta ciudad, ella también entró en la dinámica neoliberal del mercado del valor de cambio y no de su uso propiamente, pero lo hizo de manera tardía a otras ciudades latinoamericanas y colombianas.

Lo que demuestra esta forma de multicentrismo, el boom inmobiliario y el cambio de expresión de la segregación es que la globalización desarrollada bajo el modelo neoliberal ha recreado estructuras de ciudad con elementos que vienen de otros países por ejemplo, la construcción de condominios de lujo en las zonas periféricas de la ciudad en áreas rururbanas, no obstante en ciudades como Ibagué no se presenta en esas áreas. Por ello es importante llamar la atención en políticas habitacionales propias de cada ciudad respetando las normas nacionales, pero dejando de lado las tendencias mundiales que están descontextualizadas de los territorios locales (García-Granero, 2018), esto con el fin que la 
representación sea la consigna permanente por mitigar la relación de poder que existe entre el mercado inmobiliaria con la mirada cómplice del Estado hacia las personas, las cuales están en una búsqueda permanente de una distribución adecuada de recursos y de un reconocimiento recíproco (Fraser, 2008a).

Finalmente, en Ibagué ya no se concentra la población de condición socioeconómica alta en el cono de alta renta, pasó a estar por fuera de él en especial en la zona norte, centro norte y al suroriente de la ciudad; la población de condición socioeconómica baja se sigue localizando en las zonas periféricas de Ibagué en donde se planifican y construyen los proyectos VIS y VIP; con el surgimiento de los nuevos centros comerciales en el presente siglo se obtienen nuevas zonas de mercado en el centro geográfico y con ellas una tendencia al alza de los precios del suelo, que contribuyen a la propensión de adquisición de vivienda por parte de la población de nivel socioeconómico bajo hacia la periferia y por último, la forma de ciudad dual que la ciudad presentaba bajo el patrón tradicional de la segregación cambia, a una forma de expansión residencial discontinua bajo la nueva expresión de este fenómeno.

\section{Conclusiones}

El papel del Estado se cuestiona al ser uno de los productores de segregación en la ciudad de Ibagué al permitir nuevos desarrollos inmobiliarios urbanos que no promueven la integración social y espacial a la que tienen derecho todos los grupos poblacionales. Por lo tanto, junto a los promotores inmobiliarios han transformado la estructura urbana de la ciudad de una compacta a una fragmentaria y de manera paralela un fenómeno que pasó de ser de patrón tradicional a uno nuevo. La particularidad de la ciudad es que aún no se desarrollan enclaves en la periferia con tendencia al borde urbano como sucede en otras ciudades a nivel nacional, es un patrón de distribución propio de la ciudad pero que no la hace diferente en los niveles de segregación que manifiestan que existe y a una microescala bajo un modelo fragmentario y que las personas no se localizan de manera aleatoria en la ciudad.

Lo que significa que en Ibagué hay una posible heterogeneidad de los grupos que les permite una mezcla espacial más no social. Por consiguiente, las escalas de grado división y densidad explican también el nuevo patrón de segregación y dan paso al debate de las escalas de valor en el que al Estado le debe de importar representar a los grupos poblacionales excluidos para garantizarles una redistribución y reconocimiento en la ciudad.

Por lo anterior, las expresiones de la segregación que se identificaron, lo que permitieron evidenciar es el cambio que se dio en la estructura urbana de Ibagué. Por ello, desde la nueva expresión de la segregación lo que ocasiona es el rompimiento de la ciudad colonial hasta llegar a una ciudad fragmentaria en el presente siglo. Este panorama, llama la atención en la generación de políticas habitacionales y urbanas en la que se tenga en cuenta que existe un nuevo patrón de segregación que se debe afrontar y dejar atrás la creencia tradicional que si los grupos poblaciones se encuentran geográficamente cada vez más cerca, entonces la segregación residencial se verá disminuida.

Finalmente, estudiar este fenómeno complejo en una ciudad intermedia como Ibagué, la cual tiene sus propias vicisitudes por su localización geográfica, por su tamaño, por un conflicto armado (característico a nivel nacional), entre otras; precisa de manera perentoria asumir la segregación como fenómeno socioespacial en el que la escala geográfica y el periodo de tiempo en el que se estudie, juegan un papel importante para analizarlo y para procurar por soluciones para su mitigación.

\section{Agradecimientos}

Proyecto "Formación de Talento Humano de Alto Nivel" aprobado por el Fondo de Ciencia, Tecnología e Innovación (CTeI) del Sistema General de Regalías (SGR) - BPIN 2013000100103, Gobernación del Tolima y Universidad del Tolima, Colombia. 


\section{Bibliografía}

Alcaldía de Ibagué. (2008). Estudio de caracterización e identificación de asentamientos humanos precarios en la ciudad de Ibagué. Ibagué.

Bähr, J., \& Borsdorf, A. (2005). La Ciudad Latinoamericana: La construcción de un modelo. Vigencia y perspectivas. Revista de Ciudad, Urbanismo y Paisaje Ur[b]Es, 207-221.

Borsdorf, A. (2003). Cómo modelar el desarrollo y la dinámica de la ciudada latinoamericana. Eure, XXIX(68), 37-49.

Borsdorf, A., Hidalgo, R., \& Vidal-Koppmann, S. (2016). Social segregation and gated communities in Santiago de Chile and Buenos Aires. A comparison. Habitat International, 54, 18-27. https://doi.org/10.1016/j.habitatint.2015.11.033

Bourdieu, P. (1999). Efectos de Lugar. In La Miseria del Mundo (pp. 119-124). Buenos Aires: Fondo de Cultura Económica.

Ceballos, O. L., Saldarriaga, A., \& Tarchópulos, D. (2008). Vivienda Social en Colombia. Una mirada desde su Legislación 1918-2005. (O. L. Ceballos Ramos, Ed.). Bogotá D.C: Pontifica Universidad Javeriana.

Ciccolella, P., \& Vecslir, L. (2010). Nuevos espacios del terciario y transformación metropolitana en Buenos Aires. In XI Seminario Internacional RII y IV Taller de Editores RIER Universidad Nacional de Cuyo, 26-30 de octubre de 2010 Grupo (pp. 26-30). Universidad Nacional de Cuyo, 26-30 de octubre de 2010.

Clavijo, C. (2009). Crecimiento Urbano y Ordenamiento Territorial en Ibagué 1997- 2007 Cuatro Casos de la Provisión de Vivienda Nueva 1) El Vergel, 2) Los Tunjos, 3) Vasconia y Nueva Castilla y 4) El Oasis y San Gelato. Universidad Nacional de Colombia. https://doi.org/10.1017/CB09781107415324.004

Contreras, Y. (2017). De los "gentries" a los precarios urbanos . Los nuevos residentes del centro del Santiago. Eure, 43(1288), 115-141.

Cortés, J. (2010). La ciudad cautiva. Control y vigilancia en el espacio urbano. Madrid: Akal S.A.

De Mattos, C. (2012). Notas sobre una falsa disyuntiva: redensificación de las áreas centrales versus dispersión urbana. Tendencias recientes, evidencia empírica. Instituto de Estudios Urbanos y territoriales.

De Queiroz, L. (2003). Segregação Residencial e Políticas Públicas: Análise do Espaço Social da Cidade na Gestão do Território. Cidades - Comunidades e Territórios, (6), 33-50. Retrieved from http://cidades.dinamiacet.iscteiul.pt/index.php/CCT/login?source=/index.php/CCT/article/view/178/165

Espinosa, M. A. (1992). Crecimiento Urbano de Ibagué 1938-1989. Universidad Pedagógica y Tecnológica de Colombia.

Fernández de Córdova, G. (1994). El Estado: ¿Reproductor de la segregación urbana en Lima Metroplitana? Pontificia universidad Católica de Chile.

Francel, A. (2017). El Plan Urbanístico Ibagué Futuro 1935. Ibagué: Sello Editorial Universidad del Tolima.

Fraser, N. (2008a). Escalas de Justicia. Barcelona: Herder Editorial, S. L., Barcelona.

Fraser, N. (2008b). La justicia social en la era de la política de identidad: redistribución, reconocimiento y participación. Revista de Trabajo, 4(6), 83-99.

García-Granero, M. (2018). Injusticias de género en tiempos de neoliberalismo. El planteamiento de Nancy Fraser. $207-223$.

González, J. (2003). Cambios en la Vivienda Urbana en Ibagué: Un Problema de Historia de las Mentalidades. Revista Electrónica De Geografía Y Ciencias Sociales, 7(146).

González, J. (2006). De la ciudad al territorio. La configuración del espacio urbano en Ibagué 1886-1986. Ibagué: Aquelarre. Centro Cultural Universidad del Tolima.

Hidalgo, R. (2007). ¿Se acabó el suelo en la gran ciudad? Las nuevas periferias metropolitanas de lavivienda social en Santiago de Chile. Eure, XXXIII, 57-75.

Janoschka, M. (2002). El nuevo modelo de la ciudad Latinoamericana: Fragmentación y privatiación. EURE, XXVIII, 1129. https://doi.org/10.4067/S0250-71612002008500002

Lacarrieu, M. (2007). La “insoportable levedad” de lo urbano. Eure, 33(99), 47-64. https://doi.org/10.4067/S025071612007000200005 
López, A. (2018). Segregación socioespacial bajo el nuevo modelo de ciudad en América Latina. Características, perspectivas e implicaciones. Revista Hallazgos, 15(30), 99-124.

Méndez, R. (2001). Transformaciones económicas y reorganización territorial en la región metropolitana de Madrid. EURE, XXVII, 141-161.

Prévôt-Schapira, M. (2000). Segregación, fragmentación, secesión. Hacia una nueva geografía social en la aglomeración de Buenos Aires. Economía, Sociedad y Territorio, 2(7), 405-431.

Rivera, J. (2016). Análisis geo-histórico del proceso de urbanización en América del Sur: de la ciudad indoamericana a la ciudad neoliberal. Perspectiva Geográfica, 21(1), 151-178.

Ruiz-Tagle, J., \& López, E. (2014). El estudio de la segregación residencial en Santiago de Chile : revisión crítica de algunos problemas metodológicos y conceptuales. EURE, 40(119), 25-48.

Sabatini, F. (2003). La segregación social del espacio en las ciudades de América Latina. Banco Interamericano de Desarrollo, 1-41. Retrieved from http://www.iadb.org.uy/sds/doc/SOCSabatiniSegregacion.pdf

Sabatini, F., \& Cáceres, G. (2005). Relación entre Promoción Inmobiliaria y Segregación Residencial: Giros Insospechados de la Ciudad Latinoaméricana. Lincoln Institute of Land Policy. https://doi.org/10.1017/CB09781107415324.004

Sabatini, F., Cáceres, G., \& Cerda, J. (2001). Segregación residencial en las principales ciudades chilenas: Tendencias de las tres últimas décadas y posibles cursos de acción. EURE, XXVII. https://doi.org/10.4067/S025071612001008200002

Saraví, G. (2008). Mundos aislados: segregación urbana y desigualdad en la ciudad de México. EURE (Santiago), 34(103), 93-110. https://doi.org/10.4067/S0250-71612008000300005

Sassen, S. (2015). Expulsiones. Brutalidad y Complejidad en la Economía Global (Primera Ed). Buenos Aires: Katz Editores.

Schteingart, M. (2001). La división social del espacio en las ciudades. Perfiles Latinoamericanos, 13-31.

Secretaría de Planeación. (2014). Observatorio Habitacional 2014. Ibagué.

Torres, C., Gaviria, A., Zuñiga, D., Vargas, J., Nieto, D., \& Bustos, S. (2009). Ciudad informal colombiana Barrios construidos por la gente. Universidad Nacional de Colombia.

Torres, H., Marques, E., Ferreira, M., \& Bitar, S. (2003). Pobreza e espaço: padrões de segregação em São Paulo. Estudos Avançados, 17(47), 97-128. https://doi.org/10.1590/S0103-40142003000100006

Uribe, C. (2008). Estratificación social en Bogotá: de la política pública a la dinámica de la segregación social. Universitas Humanística, 65, 139-171. https://doi.org/10.1785/gssrl.79.2.194

White, M. (1983). The measurement of spatial segregation. American Journal of Sociology, 1008-1018.

Editor responsable: Rodrigo Firmino

Recibido el 08 mar. 2020

Aprobado el 15 oct. 2020 